ARTICLE

https://doi.org/10.1038/s41467-020-19866-8

\title{
Non-invasive single-cell morphometry in living bacterial biofilms
}

\author{
Mingxing Zhang (10 1,4, Ji Zhang ${ }^{1,4}$, Yibo Wang ${ }^{1,4}$, Jie Wang (1) ${ }^{2}$, Alecia M. Achimovich ${ }^{3}$, Scott T. Acton ${ }^{2} \&$
} Andreas Gahlmann (1D) 1,3凶

Fluorescence microscopy enables spatial and temporal measurements of live cells and celIular communities. However, this potential has not yet been fully realized for investigations of individual cell behaviors and phenotypic changes in dense, three-dimensional (3D) bacterial biofilms. Accurate cell detection and cellular shape measurement in densely packed biofilms are challenging because of the limited resolution and low signal to background ratios (SBRs) in fluorescence microscopy images. In this work, we present Bacterial Cell Morphometry 3D $(B C M 3 D)$, an image analysis workflow that combines deep learning with mathematical image analysis to accurately segment and classify single bacterial cells in 3D fluorescence images. In $B C M 3 D$, deep convolutional neural networks (CNNs) are trained using simulated biofilm images with experimentally realistic SBRs, cell densities, labeling methods, and cell shapes. We systematically evaluate the segmentation accuracy of $B C M 3 D$ using both simulated and experimental images. Compared to state-of-the-art bacterial cell segmentation approaches, $B C M 3 D$ consistently achieves higher segmentation accuracy and further enables automated morphometric cell classifications in multi-population biofilms.

\footnotetext{
${ }^{1}$ Department of Chemistry, University of Virginia, Charlottesville, VA, USA. ${ }^{2}$ Department of Electrical \& Computer Engineering, University of Virginia, Charlottesville, VA, USA. ${ }^{3}$ Department of Molecular Physiology \& Biological Physics, University of Virginia School of Medicine, Charlottesville, VA, USA.

${ }^{4}$ These authors contributed equally: Mingxing Zhang, Ji Zhang, Yibo Wang. ${ }_{\text {email: agahlmann@virginia.edu }}$
} 
B iofilms are multicellular communities of microorganisms that grow on biotic or abiotic surfaces ${ }^{1-4}$. In addition to cellular biomass, biofilms also contain an extracellular matrix (ECM), which is composed of polysaccharides, DNA, and proteins. Individual cells in biofilms interact with other cells, the ECM, or with the substrate surface, and the sum total of these interactions provide bacterial biofilms with emergent functional capabilities beyond those of individual cells. For example, biofilms are orders of magnitude more tolerant toward physical, chemical, and biological stressors, including antibiotic treatments and immune system clearance ${ }^{1,2,5-8}$. Understanding how such capabilities emerge from the coordination of individual cell behaviors requires imaging technologies capable of resolving and simultaneous tracking of individual bacterial cells in 3D biofilms.

Live-cell-compatible imaging technologies, such as optical microscopy, can reveal the spatial and temporal context that affects cellular behaviors. However, conventional imaging modalities are not able to resolve individual cells within thick 3D biofilms over extended periods of time. For example, the diffraction-limited lateral $x, y$-resolution $(\sim 230 \mathrm{~nm})$ of a confocal fluorescence microscope is barely sufficient to resolve bacterial cells positioned next to each other on flat glass coverslips. Even worse, the diffraction-limited axial $z$-resolution $(570 \mathrm{~nm})$ is comparable to the size of a single bacterial cell, so that densely packed cells become unresolvable in the axial $z$-dimension ${ }^{9,10}$. Notable exceptions include loose biofilms (low cell density), spherical cell shapes ${ }^{11,12}$, and mutant Vibrio cholera biofilms, in which cell-cell spacing is increased through the overproduction of ECM materials ${ }^{13-15}$. While single-cell-resolved images have been obtained in such special situations, conventional optical microscopy modalities are not generally capable to accurately resolve and quantitatively track individual cells in dense 3D biofilms.

While super-resolution derivatives of confocal microscopy, known as image scanning microscopy ${ }^{16}$, can improve spatial resolution, a perhaps more important limitation for long-term live-cell imaging is photodamage to the specimen (phototoxicity) and to the fluorophores used for labeling (photobleaching) ${ }^{17-19}$. In confocal microscopy-based approaches, undesired out-of-focus fluorescence emission is filtered out by confocal pinholes to yield optically sectioned images with high contrast, i.e., high signal-tobackground ratios (SBRs). However, repeated illumination of outof-focus regions during laser scanning and high light intensities at the focal volume result in rapid photobleaching of fluorophores and unacceptable phototoxicity for light sensitive specimens ${ }^{17-19}$. In fact, confocal fluorescence microscopy (as well as its superresolution derivatives) uses illumination light intensities that are two to three orders of magnitude higher than the light intensities under which life has evolved ${ }^{18}$. The high rates of phototoxicity and photobleaching make confocal-based microscopy unsuitable for high frame-rate time-lapse imaging of living specimens over many hours and days ${ }^{14,15,17,20,21}$.

In recent years, light sheet-based fluorescence excitation and imaging approaches have been developed to overcome the drawbacks of confocal microscopy. Among these, lattice light sheet microscopy (LLSM) ${ }^{18,19}$ and field synthesis variants thereof ${ }^{22}$, axially swept light sheet microscopy ${ }^{23,24}$, dual-view light sheet microscopy 25,26 , and single-objective oblique plane light sheet microscopy ${ }^{27-31}$ now combine excellent $3 \mathrm{D}$ spatial resolution with fast temporal resolution and low phototoxicity at levels that cannot be matched by confocal microscopy. Specifically, light sheet-based microscopy approaches can operate at illumination intensities that are below the levels of cellular phototoxicity, even for notoriously light sensitive specimens, and reduce fluorophore photobleaching by $20-50$ times compared to confocal microscopy, while maintaining comparable spatial resolution and contrast/SBR ${ }^{18,28}$.
An additional challenge in high-resolution biofilm imaging is data quantification. Even if sufficient resolution and high SBRs can be achieved to visually discern, i.e., qualitatively resolve individual cells, robust computational algorithms are still needed for automated cell segmentation and quantitative cell tracking. Toward this goal, image processing approaches based on the watershed technique and intensity thresholding have been developed over the years for single-cell segmentation in bacterial biofilms ${ }^{13-15,21}$. The broad applicability of watershed- and threshold-based image processing algorithms is however limited, because these algorithms require manual optimization of many user-selected parameters. Even with optimal parameters, watershed- and threshold-based image processing methods often produce suboptimal segmentation results, especially when cell densities are high, when SBRs are low, and when cellular fluorescence intensities are not uniform across the cytosol or the cell surface. To overcome the drawbacks of traditional mathematical image processing approaches, automated solutions based on supervised training of deep convolutional neural networks (CNNs) have been used in recent years with great success for a wide range of problems in biomedical image analysis ${ }^{32}$.

Here, we present Bacterial Cell Morphometry 3D $(B C M 3 D)^{33}$, a generally applicable workflow for single-cell segmentation and shape determination in high-resolution 3D images of bacterial biofilms. $B C M 3 D$ uses $C N N s$, in silico-trained with computationally simulated biofilm images, in combination with mathematical image analysis to achieve accurate single-cell segmentation in $3 \mathrm{D}$. The CNNs employed in $B C M 3 D$ are based on the $3 \mathrm{D} \mathrm{U}-\mathrm{Net}$ architecture and training strategy, which has achieved excellent performance in biomedical data analysis benchmark tests ${ }^{32}$. The mathematical image analysis modules of $B C M 3 D$ enable post-processing of the CNN results to further improve the segmentation accuracy. We establish that experimental bacterial biofilms images, acquired by LLSM, can be successfully segmented using CNNs trained with computationally simulated biofilm images, for which the ground truth voxel-level annotation maps are known accurately and precisely. By systematically evaluating the performance of $B C M 3 D$ for a range of SBRs, cell densities, and cell shapes, we find that voxel-level segmentation accuracies of $>80 \%$, as well as cell counting accuracies of $>90 \%$, can be robustly achieved. BCM3D consistently outperforms previously reported image segmentation approaches that rely exclusively on conventional image processing approaches. $B C M 3 D$ also achieves higher segmentation accuracy on experimental 3D biofilm data than Cellpose ${ }^{34}$, a state-of-the-art, CNN-based, generalist algorithm for cell segmentation, and the algorithm used by Hartmann et al. ${ }^{15}$, a specialized algorithm designed for bacterial cell segmentation based on traditional mathematical image processing. We expect that $B C M 3 D$, and $C N N$-based single-cell segmentation approaches in general, combined with noninvasive light sheet-based fluorescence microscopy will enable accurate cell tracking over time in dense 3D biofilms. This capability will launch a new era for bacterial biofilm research, in which the emergent properties of microbial populations can be studied in terms of the fully resolved behavioral phenotypes of individual cells.

\section{Results}

Cell segmentation by thresholding CNN confidence maps. CNNs have been shown to perform well on pixel-level classification tasks for both $2 \mathrm{D}$ and $3 \mathrm{D}$ data ${ }^{35,36}$. Bacterial biofilms, however, present a unique challenge in this context. The cell shapes to be segmented are densely packed and barely resolvable even with the highest resolution optical microscopes. In addition, living biofilms in fluorescence microscopes can only be imaged 

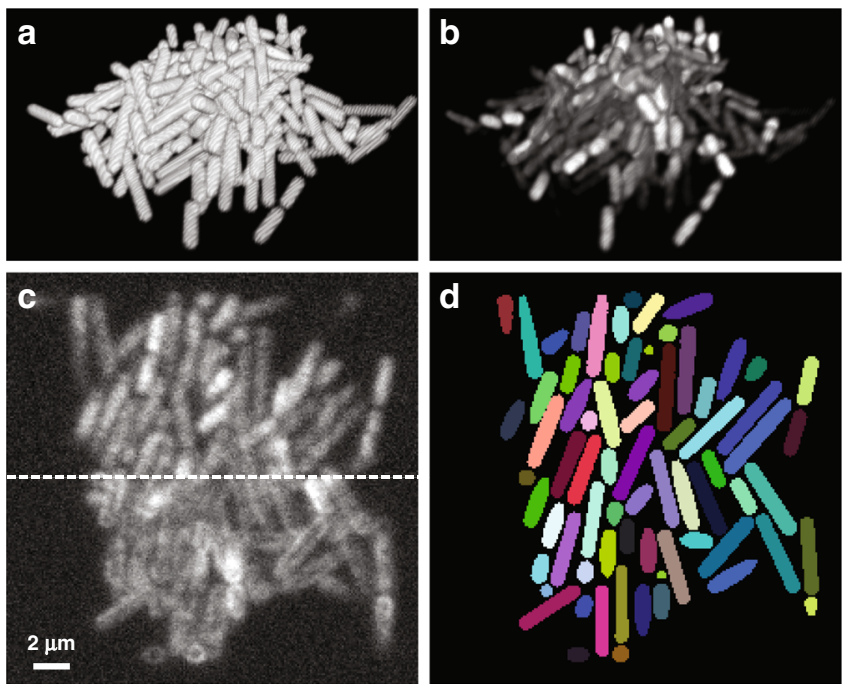

Fig. 1 Simulation of fluorescent biofilms images and annotation maps used for CNN training. a Representative cell arrangements obtained by CellModeller. Due to the stochastic nature of biofilm growth, different cell arrangements are obtained in each new simulation. However, cell density is reproducible for each new simulated biofilm (typically $N=10$ different biofilm simulations are used for CNN training, see "Methods" section). b Simulated 3D fluorescence image based on the cell arrangements in $\mathbf{a}$. c $X Y$ slice through the 3D simulated fluorescence image in $\mathbf{b}$ (upper panel shows cells expressing cytosolic fluorescent proteins, lower panel shows cells stained with membrane-intercalating dyes). d Ground truth cell

arrangements giving rise to the image shown in c. Voxels are displayed as black (background), or in different colors (indicating different cells).

with low laser intensities to ameliorate phototoxicity and photobleaching concerns. Unfortunately, low-intensity fluorescence excitation also reduces the SBR in the acquired images. So far, it remains unclear to what extent single-cell segmentation approaches can accurately identify and delineate cell shapes in bacterial biofilm images obtained under low-intensity illumination conditions. To address this question, we implemented an in silico CNN training strategy and systematically evaluated its voxel-level classification (cell morphometry) and cell counting accuracies, using simulated biofilm images with cell densities and SBRs similar to those encountered in experimental data (see "Methods" section).

We compared two commonly used cell labeling approaches, namely genetic labeling through the expression of cell-internal fluorescent proteins and staining of the cell membranes using fluorescent dyes (Fig. 1). For both labeling approaches, voxel-level segmentation and cell counting accuracies, obtained by thresholding CNN confidence maps (see "Methods" section), depend mostly on cell density, whereas the SBR plays a less important role (Fig. 2a-f). For cell-internal labeling, SBRs of $>1.7$ and cell densities of $<60 \%$ consistently produce voxel-level classification accuracies of $>80 \%$ and cell counting accuracies of $>95 \%$. On the other hand, SBRs of $<1.7$ and cell densities of $>60 \%$ lead to lower segmentation accuracies. While lower segmentation accuracies are expected for higher cell densities and lower SBRs, the sharp drop-offs observed here may indicate a fundamental performance limitation of the CNNs employed. Still, the voxel-level classification and cell counting accuracies consistently surpass previous approaches for bacterial cell segmentation for commonly encountered cell densities and SBRs. Specifically, the cell counting accuracies obtained by Hartmann et al. ${ }^{15}$, Seg3 ${ }^{37}$, and Yan et al. ${ }^{13}$ quickly drop to zero as a function of increasing
Intersection-over-Union (IoU) matching threshold (a quantitative measure of cell shape similarity relative to the ground truth, see "Methods" section), indicating that cell shapes are not accurately estimated by conventional image processing approaches (Fig. 2g-i). We also evaluated the segmentation accuracy of Cellpose, a recently developed, CNN-based cellular segmentation algorithm ${ }^{34}$. The segmentation accuracy of Cellpose is comparable or superior to the best-performing conventional image processing approaches-a considerable achievement given that Cellpose was trained primarily on images of eukaryotic cells. However, being a pretrained generalist model, the segmentation accuracy of Cellpose is lower than the accuracy achieved by the specialist in silico-trained CNNs of $B C M 3 D$, which were trained specifically for $3 \mathrm{D}$ bacterial biofilm segmentation. Overall, the cell counting accuracies obtained by $B C M 3 D$ are higher than other methods and remain higher even for IoU matching thresholds larger than 0.5 , indicating that cell shapes are more accurately estimated by the in silico-trained CNNs.

The accuracies of single-cell shape estimation and cell counting are predominantly affected by cell density. The variation is more prominent for membrane-stained cells, because intercellular fluorescence intensity minima are less pronounced, when cell membranes are labeled and cells physically contact each other (red arrow in Fig. 2c, f). By contrast, intracellular fluorophores produce the highest intensities at the cell center, so that the gaps between cells are more readily resolvable. Also noteworthy is the sharp drop-off in segmentation accuracies for SBRs of $<1.7$ for all cases. In such low SBR regimes, fluorescence signals of the cells become too difficult to be distinguished from the background. As a result, the CNNs falsely identify random noisy patterns in the background as cells. In addition, thresholding of the CNN confidence maps often yields connected voxel clusters that contain multiple bacterial cells. False identification and incomplete delineation of cells cause the pronounced decrease in segmentation accuracy for SBRs of $<1.7$.

Post-processing of CNN confidence maps. To better identify individual cells in low SBR and high cell density datasets, we developed a graph-based post-processing module (see "Methods" section) that takes advantage of the fact that bacterial cell shapes are highly conserved for a given species. Briefly, we transformed the $\mathrm{CNN}$ cell interior confidence maps into $3 \mathrm{D}$ point cloud data that trace out the central axes of individual cells. This transformation was achieved by medial axis extraction using sizeconstrained inscribed spheres ${ }^{38}$ (Supplementary Fig. S1a-c). Single-cell axes are then identified as linearly clustered data points by linear cuts (LCuts) - a graph-based data clustering method designed to detect linearly oriented groups of points ${ }^{39}$. The soidentified single-cell axes are then mapped back onto the original segmentation volumes to obtain estimates of the 3D positions, shapes, and orientations of the now separated cells (Supplementary Fig. S1d).

Post-processing with LCuts takes advantage of a priori knowledge about expected bacterial cell sizes by removing erroneously segmented volumes that are significantly smaller than the expected value and by splitting incompletely segmented volumes representing fused cells. Improvements in cell counting accuracy of up to $15 \%$ and $36 \%$ are observed for cells labeled with cytosolic fluorophores (Fig. $3 \mathrm{a}-\mathrm{c}$ and Supplementary Fig. S2) and membrane-localized fluorophores (Fig. 3d-f), respectively. The more substantial improvement for membrane-stained cells is due to fact that CNNs trained on membrane-stained cells are more prone to erroneously identifying speckled background noise as fluorescence signals in low SBR images. In addition, membrane-intercalating 
a

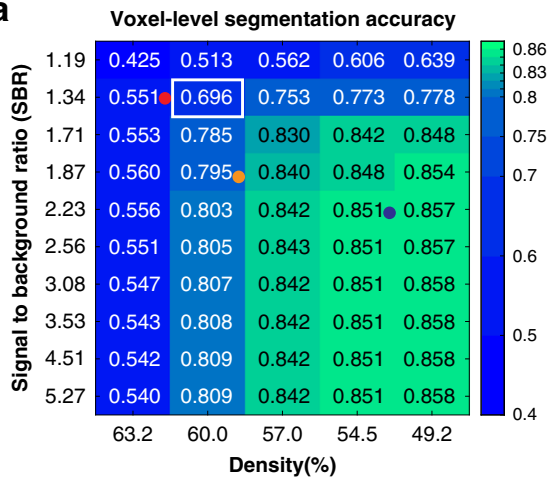

d

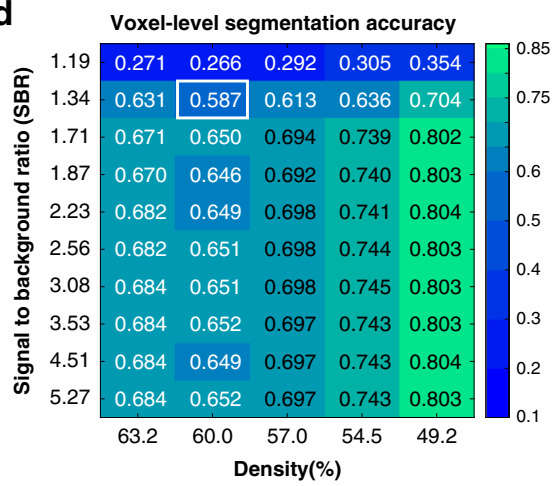

b

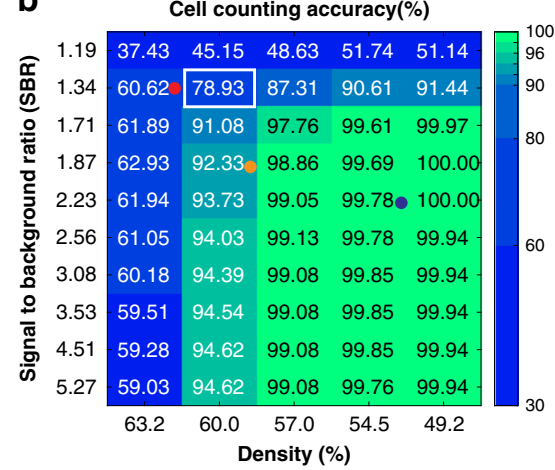

e
C Cytosolic fluorescence

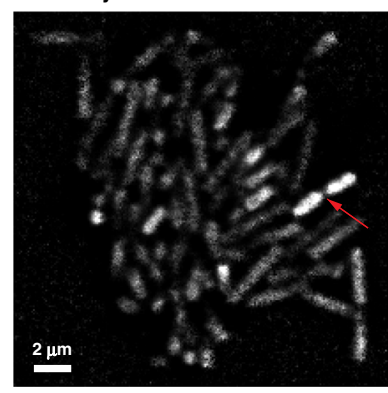

f Cell membrane fluorescence

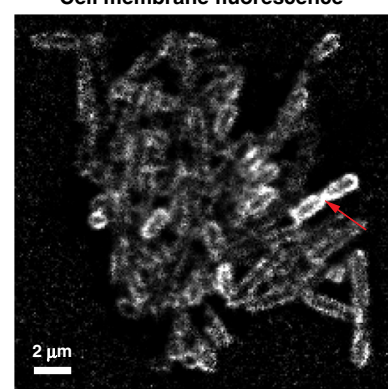

g

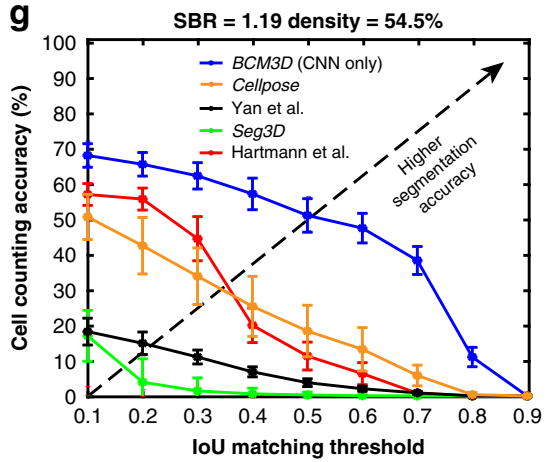

h

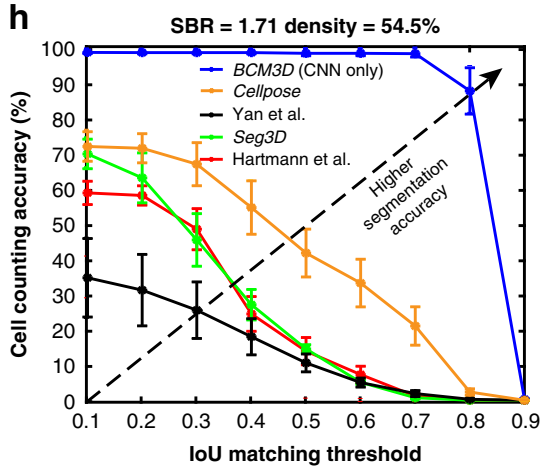

i

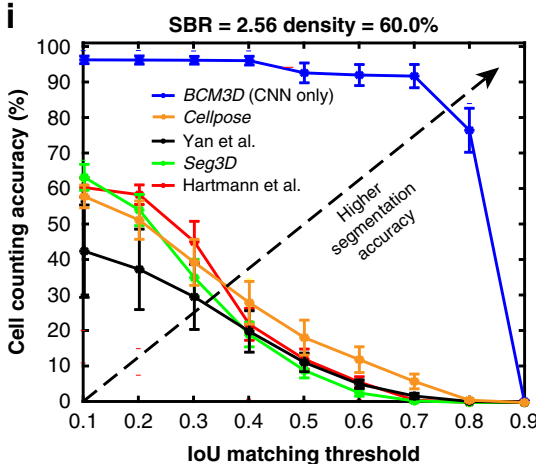

Fig. 2 Performance of BCM3D using in silico-trained CNNs only on previously unseen simulated biofilm images. a The voxel-level segmentation accuracy quantifies whether each voxel has been assigned to the correct class ("cell interior", "cell boundary", or "background"). Solid circles represent the maximum local density and average SBRs encountered in experimental datasets (red, orange, and blue: E. coli expressing GFP). b The cell counting accuracy (using an loU matching threshold of 0.5 for each segmented object, see "Methods" section) averaged over $N=10$ replicate datasets for cells labeled with cytosolic fluorophores. c Example image of cells labeled with cytosolic fluorophores (cell density $=60.0 \%$, SBR $=1.34$, indicated by white rectangle in $\mathbf{a}$ and $\mathbf{b}$. Similar images were generated $N=10$ times with different cell arrangements). d Voxel-level segmentation accuracy and e cell counting accuracy averaged over $N=10$ replicate datasets for cells labeled with membrane-localized fluorophores. $\mathbf{f}$ Example image of cells labeled with membrane-localized fluorophores (cell density $=60.0 \%, S B R=1.34$, indicated by white rectangles in $\mathbf{d}$ and $\mathbf{e}$. Similar images were generated $N=10$ times with different cell arrangements). The red arrows indicate a close cell-to-cell contact point. $\mathbf{g}$-i Comparison of segmentation accuracies achieved by conventional segmentation approaches (Hartmann et al., Seg3D, Yan et al.), Cellpose, and BCM3D (only using in silico-trained CNNs). Three simulated datasets (cytosolic fluorophores) with different SBRs and cell densities are shown. Segmentation accuracy is parameterized in terms of cell counting accuracy ( $y$-axis) and loU matching threshold ( $x$-axis, a measure of cell shape estimation accuracy). Each data point is the average of $N=10$ independent biofilm images. Data are presented as mean values \pm one standard deviation indicated by error bars. Curves approaching the upper right-hand corner indicate higher overall segmentation accuracy, as indicated by the dashed arrows. Source data are provided as a Source data file for Fig. $2 \mathrm{~g}$, h, i.

fluorophores of two adjacent cells are in close proximity, making it difficult to resolve fluorescence signals from two separate cells due to spatial signal overlap (see the red arrow, Fig. 2c, f). LCuts thus provides an important benefit in improving the cell counting accuracy to an extent not achieved by currently available thresholding- or watershed-based postprocessing algorithms (Supplementary Fig. S3).
Segmentation of experimental biofilm images. To test the performance of $B C M 3 D$ on experimentally acquired biofilm images, we acquired time-lapse images of GFP expressing E. coli biofilms every $30 \mathrm{~min}$ for $10 \mathrm{~h}$ (see "Methods" section). We then manually annotated one 2D slice in the 3D images at the $t=300,360$, and 600-min time points (see "Methods"). When referenced to these manual segmentation results, the LCuts-processed CNN outputs 

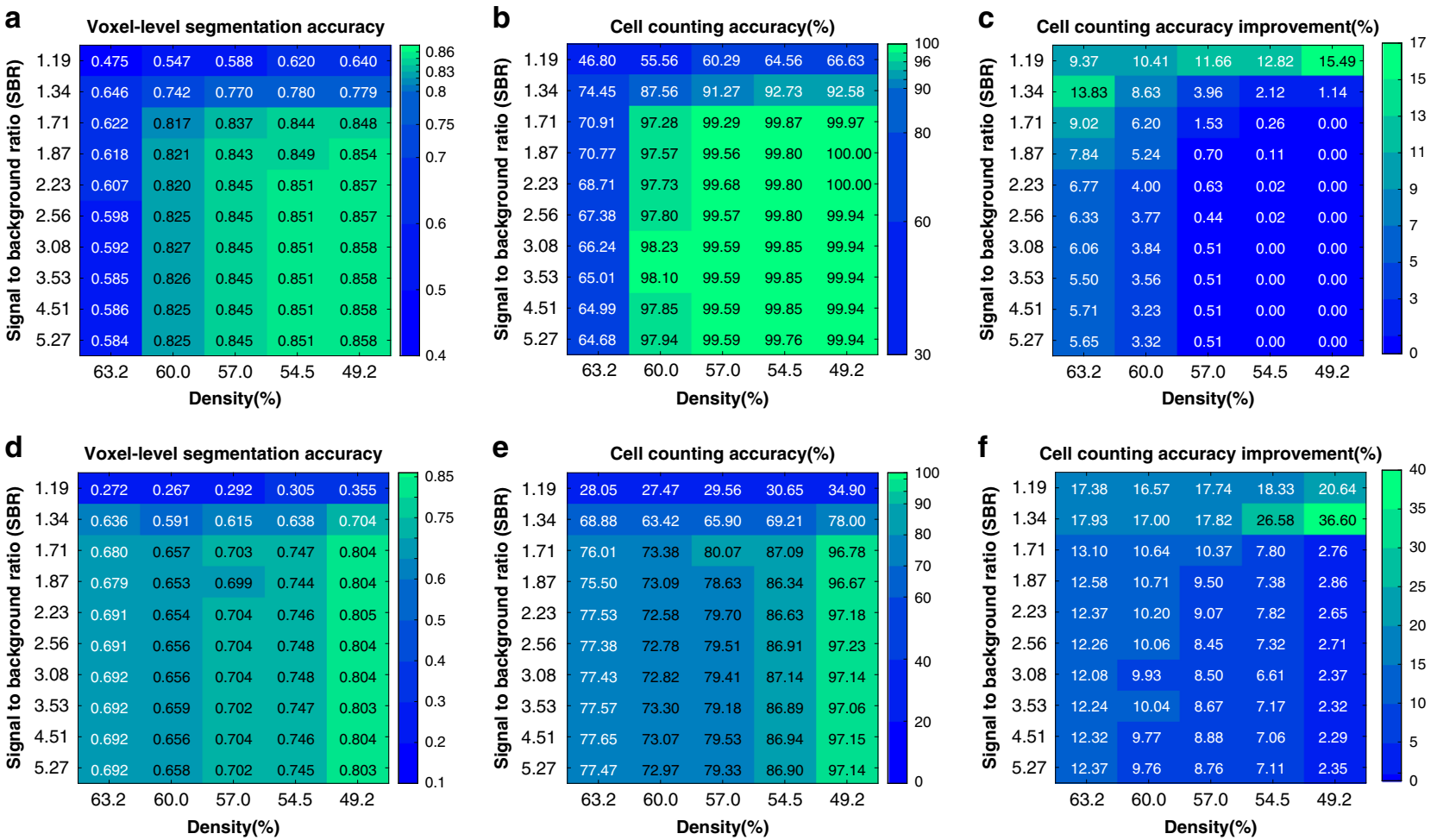

Fig. 3 Performance of BCM3D (in silico-trained CNNs and additional post-processing by LCuts) on previously unseen simulated data. a Voxel-level segmentation accuracy and $\mathbf{b}$ cell counting accuracy (using an loU matching threshold of 0.5 for each segmented object) averaged over $N=10$ replicate datasets for cells labeled with cytosolic fluorophores. c Improvement relative to silico-trained convolutional neural networks without post-processing. d Voxel-level segmentation accuracy and e cell counting accuracy averaged over $N=10$ replicate datasets for cells labeled with membrane-localized fluorophores. f Improvements relative to silico-trained convolutional neural networks without post-processing.

consistently achieved better cell counting accuracies than conventional segmentation methods (Fig. 4 and Supplementary Fig. S4). Initially, Cellpose and the Hartmann et al. algorithm outperform the in silico-trained CNNs on two out of three of the test images $(t=360$ and $600 \mathrm{~min})$, for which our in silico-trained $\mathrm{CNNs}$ struggle with undersegmentation problems. However, mathematical post-processing of the CNN outputs by LCuts corrects some of these errors, so that the integrated $B C M 3 D$ workflow achieves improved results compared to Cellpose and Hartmann et al. at each of the indicated time points. Visual inspection of the segmentation results is also informative. Cellpose accurately segments individual cells in low density regions, but suffers from oversegmentation errors in high density biofilm regions (Supplementary Fig. S4e). The Hartmann et al. algorithm provides reasonable estimates of cell positions in low and high density biofilm regions, but again struggles with cell shape estimation (Supplementary Fig. S4d and see also Fig. 2g-i). On the other hand, the integrated $B C M 3 D$ workflow (CNN + LCuts) produces biologically reasonable cell shapes regardless of cell density (Fig. 4).

We attribute the more rapid drop-off of the cell counting accuracy as a function of increasing IoU matching threshold in Fig. 4 to the following factors. First, human annotation of experimentally acquired biofilm images differs from the ground truth segmentation masks that are available for simulated data (Supplementary Fig. S5). The shape mismatches between algorithm segmented and manually annotated cell shapes (Supplementary Figs. S5 and S6) lead to a global lowering of voxel-level segmentation accuracy, and thus a more rapid dropoff of the cell counting accuracy as a function of increasing IoU matching threshold. Because bacterial cell shapes are not accurately captured by manual annotation (Supplementary Fig. S5), cell counting accuracies referenced to manual annotations should be compared only at low IoU matching thresholds (0.1-0.3, shaded grey in Fig. $4 \mathrm{a}-\mathrm{c})$, as also pointed out previously ${ }^{40}$. We also note that bacterial cells in experimental images appear motion-blurred if they are only partially immobilized, and therefore wiggle during image acquisition. Furthermore, optical aberrations and scattering effects were not included in training data simulations, which may decrease the performance of the CNNs on experimental data. Still, at IoU matching threshold $<0.3$, the cell counting accuracy of $B C M 3 D$ remains above $75 \%$, while also producing biologically reasonable cell shapes. Thus, the bacterial cell segmentation results of $B C M 3 D$ represent a substantial improvement over other approaches (Fig. 4 and Supplementary Fig. S4).

To demonstrate that $B C M 3 D$ can achieve similarly high segmentation accuracies for membrane-stained cells in different cellular arrangements, we analyzed a small patch of a Myxococcus xanthus biofilm, which was stained with the membraneintercalating dye FM4-64 (Fig. 5a). In contrast to E. coli biofilms, the submerged $M$. xanthus biofilm imaged here features cells in a mesh-like arrangement with close cell-to-cell contacts, which presents a unique challenge for $3 \mathrm{D}$ single-cell segmentation. To obtain reference data for 3D segmentation accuracy determination, we manually annotated each $x y, x z$, and $y z$ slice of an entire $3 \mathrm{D}$ image stack (Fig. 5b). When referenced to this $3 \mathrm{D}$ manual segmentation result, $B C M 3 D$ (Fig. 5c) produced cell counting accuracies above $70 \%$ at low (0.1-0.3) IoU matching thresholds, whereas segmentation results obtained by conventional image processing (Hartmann et al.) and by generalist $\mathrm{CNN}$-processing (Cellpose) produced cell counting accuracies $<50 \%$ in the same 

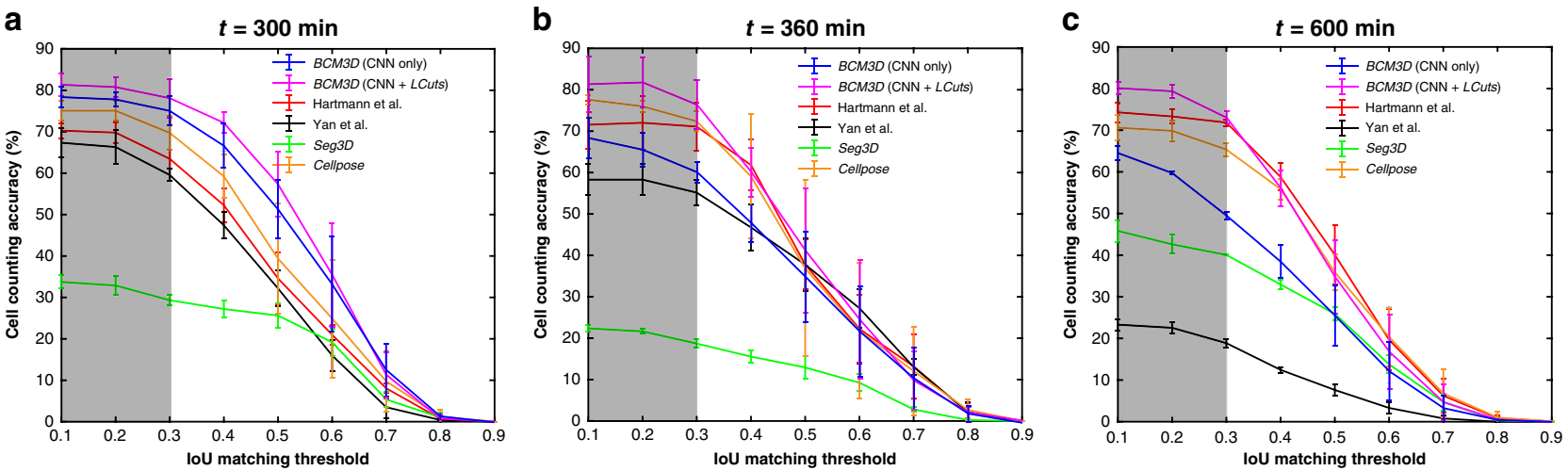

d
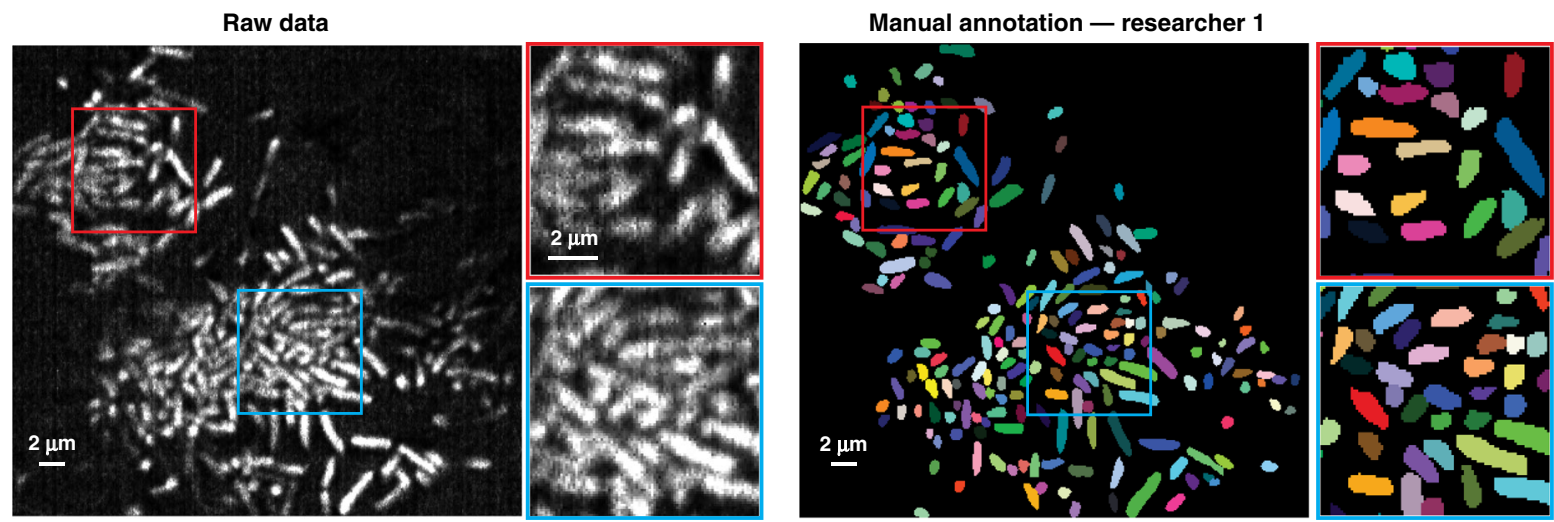

$B C M 3 D$ (CNN only)
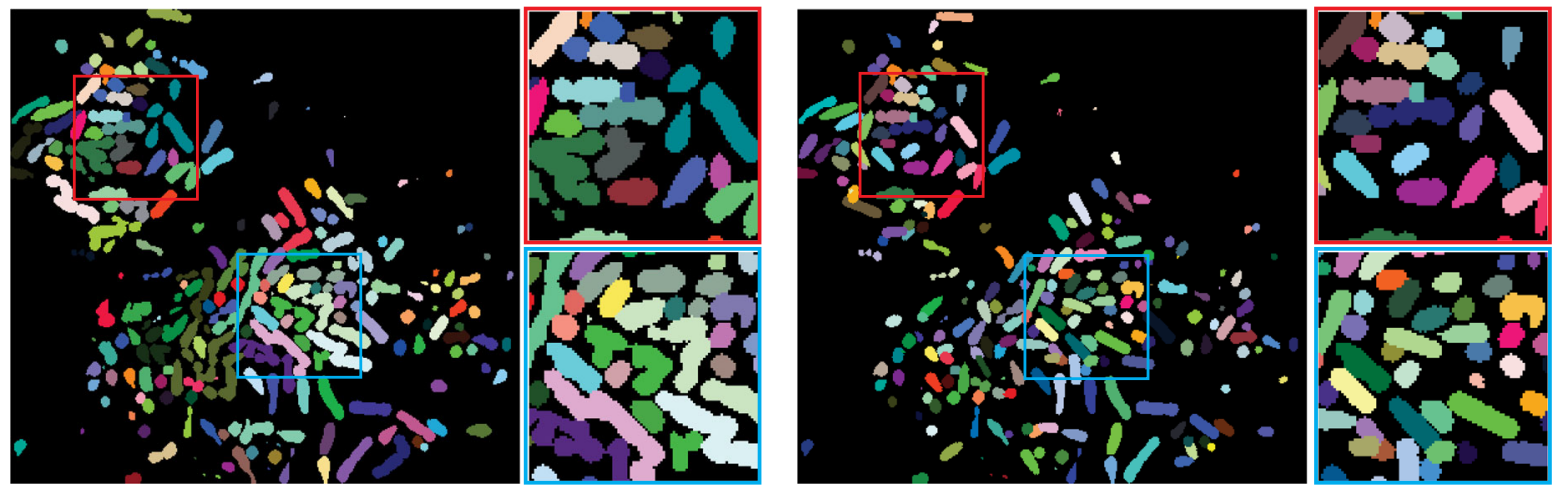

Fig. 4 Comparison of segmentation accuracies achieved by conventional segmentation approaches (Hartmann et al., Seg3D, Yan et al.), Cellpose, and BCM3D. The estimated SBRs are 2.2, 1.8, and 1.3, respectively. The estimated cell densities are 54.8\%, 59.0\%, and 64.6\%, respectively. a-c Three experimental E. coli datasets (cytosolic expression of GFP) acquired at different time points after inoculation of cells. Segmentation accuracy is parameterized in terms of cell counting accuracy ( $y$-axis) and loU matching threshold ( $x$-axis). Each data point is the average of the cell counting accuracies calculated using annotation maps traced by $N=3$ different researchers. Data are presented as mean values \pm one standard deviation indicated by error bars. Curves approaching the upper right-hand corner indicate higher overall segmentation accuracy. d Comparison of segmentation results achieved at the $t=600 \mathrm{~min}$ time point by manual annotation (shown is one of $N=3$ researchers' annotation result, the other two annotation results are shown in Supplementary Fig. S4), and by BCM3D using in silico-trained CNNs only and after further refinement of CNN outputs using LCuts. Similar results were also obtained at the $t=300$ and $t=360 \mathrm{~min}$ time points. Segmentation results of the other methods are shown in Supplementary Fig. S4. Source data are provided as a Source data file for Fig. $4 a-c$.

IoU matching threshold region (Fig. 5d). We note however that neither Cellpose nor the Hartmann et al. algorithms were specifically optimized/designed for segmenting membranestained cells. Indeed, the performance of Cellpose on this type of biofilm architecture is inferior to the results achieved using the in silico-trained CNNs of $B C M 3 D$ alone (without using LCuts post-processing). One reason might be that the pretrained, generalist Cellpose model has not been trained sufficiently on long, thin, and highly interlaced rod-shaped cells, such as those contained in a $M$. xanthus biofilm.
Morphological separation of mixed cell populations. Given the improved segmentation results obtained using $B C M 3 D$, we reasoned that the same CNNs may have additional capacity to assign segmented objects to different cell types based on subtle morphological differences in the acquired images. Differences in the imaged cell morphologies arise due to physical differences in cell shapes (e.g., spherical vs. rod-shaped cells) or due to differences in the fluorescent labeling protocols (e.g., intracellular vs. cell membrane labeling), because fluorescence microscopes simply measure the spatial distributions of fluorophores in the sample. 

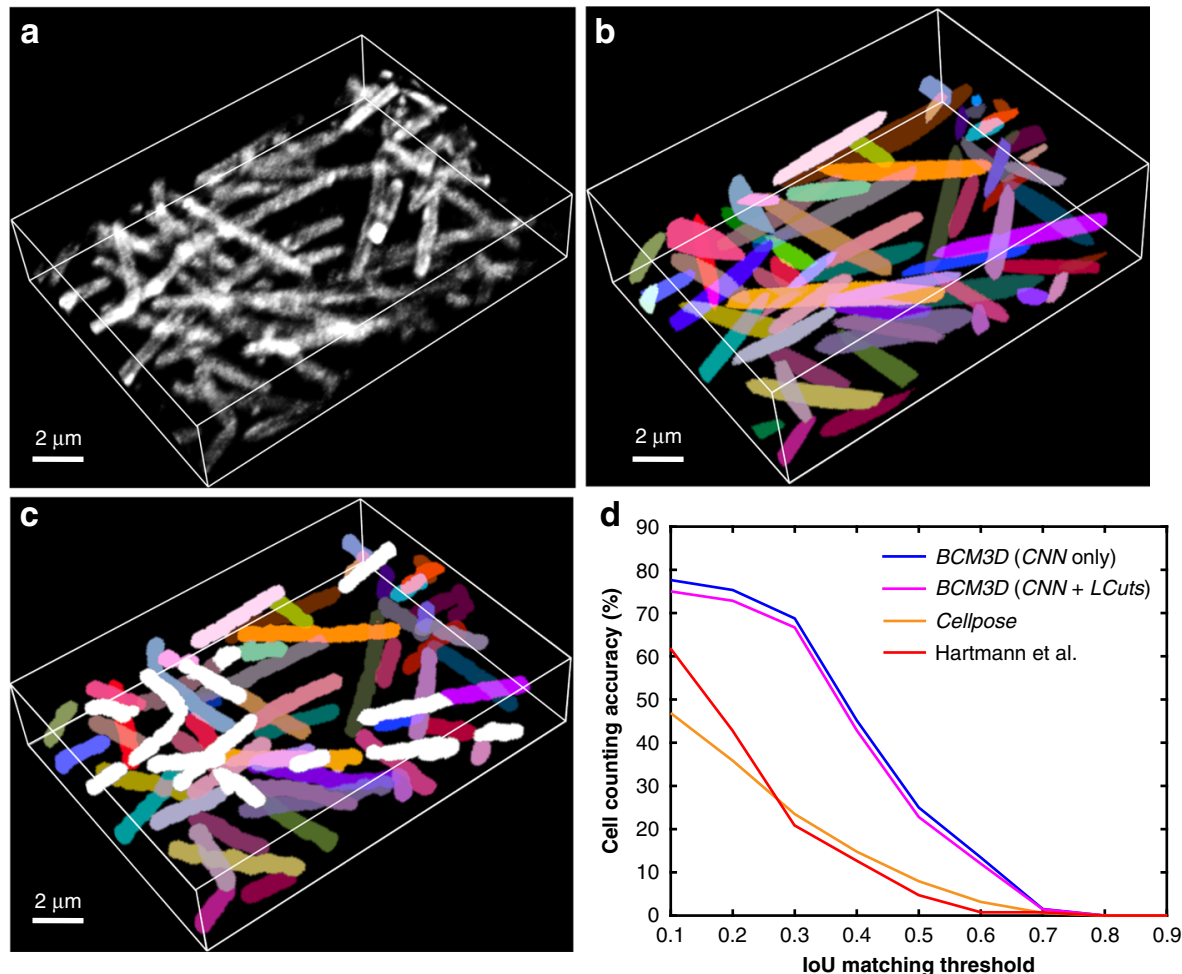

Fig. 5 3D Segmentation accuracy evaluation using $\boldsymbol{M}$. xanthus biofilm images (cell density $=\mathbf{3 6 . 2} \%$, and SBR $=\mathbf{1 . 5 8}$ ). a Maximum intensity projection of a 3D M. xanthus fluorescence image. Cells were labeled with membrane-intercalating dye, FM4-64. Similar images were obtained at $N=120$ different time points. $\mathbf{b}$ Maximum intensity projection of the manually obtained 3D segmentation result. c Maximum intensity projection of a CNN-based 3D segmentation result after LCuts post-processing. Cells that can be matched with the GT are displayed in the same colors as GT or otherwise colored in white. $\mathbf{d}$ Segmentation accuracy of compared algorithms parameterized in terms of cell counting accuracy ( $y$-axis) and loU matching threshold ( $x$-axis). Source data are provided as a Source data file for Fig. $5 \mathrm{~d}$.

The ability to separate different cell morphologies is important for the study of multispecies biofilms, where interspecies cooperation and competition dictate population-level outcomes ${ }^{3,41-48}$. Separation of differentially labeled cells is also important for the study of gene activation in response to cell-to-cell signaling ${ }^{49}$. Expression of cytosolic fluorescent proteins by transcriptional reporter strains is a widely used technique to visualize activation of a specific gene or genetic pathway in living cells. Such genetic labeling approaches can be complemented by chemical labeling approaches, e.g., using membrane-intercalating chemical dyes that help visualize cells nonspecifically or environmentally sensitive membrane dyes that provide physiological information, including membrane composition ${ }^{50,51}$, membrane organization and integrity ${ }^{52-54}$, and membrane potential ${ }^{42,55}$. Chemical and genetic labeling approaches are traditionally implemented in two different color channels. However, there are important drawbacks to using multiple colors. First and foremost, the amount of excitation light delivered is increased by the necessity to excite differently colored fluorophores, raising phototoxicity, and photobleaching concerns. Second, it takes $N$ times as along to acquire $N$-color images (unless different color channels can be acquired simultaneously), making it challenging to achieve high temporal sampling in time-lapse acquisition. For these reasons, methods that extract complementary physiological information from a single-color image are preferable.

We evaluated the ability of BCM $3 D$ to automatically segment and identify rod-shaped and spherical bacterial cells consistent, with shapes of E. coli and Staphylococcus aureus in simulated images (Supplementary Fig. S7). To segment cells in twopopulation biofilms, we trained CNNs that classify pixels into five different classes: "background", "cell interior of population 1 ", "cell boundary of population 1", "cell interior of population 2", and "cell boundary of population 2". Thresholding the CNNs confidence maps can achieve cell counting accuracies larger than $90 \%$ for both cell types independent of their population fractions (Fig. 6a). Post-processing of this result using LCuts improved the cell counting accuracy by $<0.5 \%$ on average, indicating that under-segmented cell clusters are not prevalent in this dataset.

We next evaluated the ability of $B C M 3 D$ to automatically segment and separate membrane-stained cells that express cytosolic fluorescent proteins from those that do not (Supplementary Fig. S8). Again, the cell counting accuracy is consistently above $80 \%$ for all tested mixing ratios (Fig. 6b). Finally, we applied $B C M 3 D$ to experimentally acquired biofilm images of two different $E$. coli strains. Both strains were stained by the membrane-intercalating dye FM4-64, but the second strain additionally expressed GFP (Supplementary Fig. S9). The cells were homogeneously mixed prior to mounting to randomize the spatial distribution of different cell types in the biofilm (see "Methods" section). Multiple 2D slices from the 3D image stack were manually annotated and compared with the results obtained by $B C M 3 D$. Consistent with the single-species experimental data, a cell counting accuracy of $50 \%$ is achieved for each cell type at a 0.5 IoU matching threshold and, at lower IoU matching thresholds, the counting accuracies increased to $60-70 \%$, (Fig. 6c, d). Thus, using appropriately trained CNNs in BCM $3 D$ enables automated and accurate cell type assignments based on subtle differences in cell morphologies in mixed population biofilms-a capability not available using conventional image processing methods. 

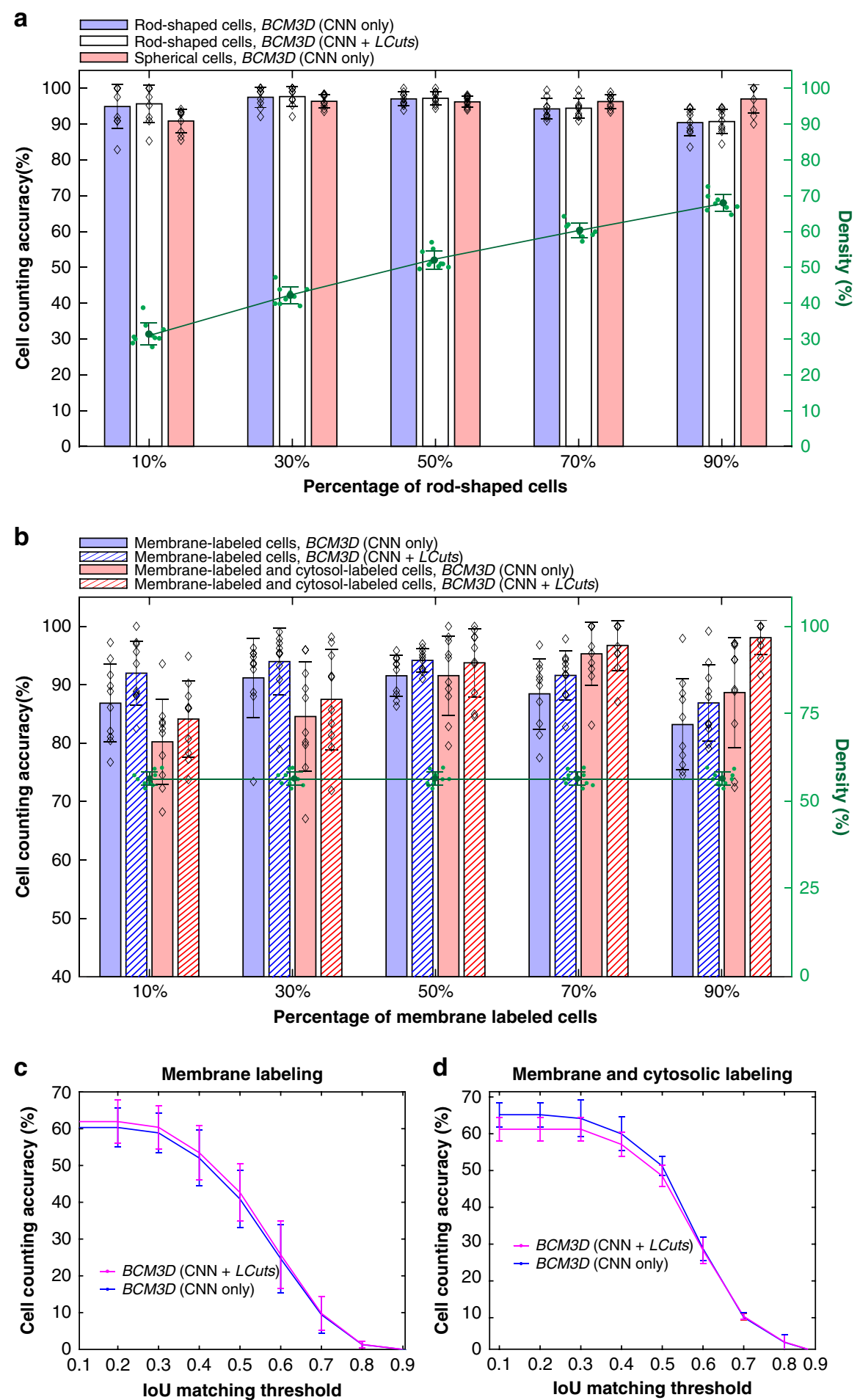

Fig. 6 Performance of BCM3D on mixed population biofilm images. a Cell counting accuracy of $B C M 3 D$ on simulated images containing different ratios of rod-shaped and spherical cells. Black diamonds represent the counting accuracy for $N=10$ independently simulated datasets. Green dots represent the cell density for each independent dataset. Error bars represent \pm one standard deviation. $\mathbf{b}$ Cell counting accuracy of BCM $3 D$ on simulated images with different ratios of membrane-labeled, and membrane-labeled and interior fluorescent protein expressing cells. Black diamonds represent the counting accuracy for $N=10$ independently simulated datasets. Green dots represent the cell density for $N=10$ independent datasets. Error bars represent \pm one standard deviation. c, $\mathbf{d}$ Cell counting accuracy of $B C M 3 D$ on experimental images of $\mathbf{c}$ membrane-labeled, and $\mathbf{d}$ membrane-labeled and interior fluorescent protein expressing $E$. coli cells (mixing ratio $~ 1: 1)$. Each data point is the average of the cell counting accuracies calculated using annotation maps traced by three different researchers $(N=3)$. Data are presented as mean values \pm one standard deviation indicated by error bars. Source data are provided as a Source data file for Fig. 6. 


\section{Discussion}

CNNs have been successful applied to many different problems in biological image analysis, but their ability to segment individual cells in 3D and time-lapse 3D bacterial biofilm images has not yet been fully explored. Here, we demonstrated a CNN-based image analysis workflow, termed $B C M 3 D$, for single-cell segmentation and shape classification (morphometry) in 3D images of bacterial biofilms. In this work, we applied $B C M 3 D$ to $3 \mathrm{D}$ images acquired by LLSM. However, $B C M 3 D$ readily generalizes to $3 \mathrm{D}$ images acquired by confocal microscopy or advanced super-resolution microscopy modalities, provided that realistic image formation models are used to simulate the training datasets. The use of simulated training data is a major advantage of $B C M 3 D$, because it overcomes inconsistencies inherent in manual dataset annotation (Supplementary Figs. S5 and S6), and thus solves the problem of obtaining sufficient amounts of accurately annotated 3D image data. The ability to use simulated training data provides needed flexibility not only in terms of the microscope platform used for imaging, but also in terms of the bacterial cell shapes that are to be segmented.

We systematically investigated the advantages and limitations of $B C M 3 D$ by evaluating both voxel- and cell-level segmentation accuracies, using simulated and experimental datasets of different cell densities and SBRs. BCM $3 D$ enabled accurate segmentation of individual cells in crowded environments and automatic assignments of individual cells to specific cell populations for most of the tested parameter space. Such capabilities are not readily available when using previously established segmentation methods that rely exclusively on conventional image and signal processing algorithms.

While $B C M 3 D$ surpasses the performance of previous approaches, we stress that further improvements are possible and, for long-term, high frame-rate time-lapse imaging experiments, absolutely needed. Our systematic analysis revealed that high cell density and low SBR datasets are particularly challenging for the CNNs used in this work. Future work will therefore focus on increasing the contrast and resolution in bacterial biofilm images. While, the use of optical super-resolution modalities can provide higher spatial resolution, such resolution improvements often come at a cost of reduced image contrast and faster photobleaching/phototoxicity. Software solutions that can process images with limited resolution and low SBRs will therefore play a tremendously important role in biological imaging. $B C M 3 D$ is a general workflow that integrates computational simulation of training data, in silico training of CNNs for a specific task or a specific cell type, and mathematical post-processing of the CNN outputs. Incorporating different training strategies and different CNNs, such as the generalist CNN used in Cellpose ${ }^{34}$, into the $B C M 3 D$ workflow will enable automated cross-validation of segmentation results when a ground truth or manual annotation map is not available. Furthermore, CNN-based image processing modules developed for contrast enhancement and denoising have also surpassed the performance of conventional methods based on mathematical signal processing ${ }^{56-59}$. Incorporating these tools into the $B C M 3 D$ workflow promises to further improve the single-cell segmentation accuracies. We anticipate that the ability to accurately identify and delineate individual cells in dense 3D biofilms will enable accurate cell tracking over long periods of time. Detailed measurements of behavioral single-cell phenotypes in larger bacterial communities will help determine how macroscopic biofilm properties, such as its mechanical cohesion/adhesion and its biochemical metabolism, emerge from the collective actions of individual bacteria.

\section{Methods}

Lattice light sheet imaging of bacterial biofilms. Fluorescence images of bacterial biofilms were acquired on a home-built LLSM. LLSM enables specimen illumination with a thin light sheet derived from $2 \mathrm{D}$ optical lattice ${ }^{18,60}$.
Here, a continuous illumination light sheet was produced by a time-averaged (dithered), square lattice pattern ${ }^{18}$, and the illumination intensity at the sample was $<1 \mathrm{~W} / \mathrm{cm}^{2}$. The submicrometer thickness of the excitation light sheet is maintained over long propagation distances $(\sim 30 \mu \mathrm{m})$, which enables optical sectioning, and thus high resolution, high contrast imaging of $3 \mathrm{D}$ specimens comparable to confocal microscopy. However, fluorophore excitation by a $2 \mathrm{D}$ light sheet reduces phototoxicity, because each excitation photon has multiple opportunities to be absorbed by fluorophores in the excitation plane and produce in-focus fluorescence. Widefield fluorescence images corresponding to each illuminated specimen plane are recorded on a sCMOS detector (Hamamatsu ORCA Flash v2). In this work, 3D biofilm images were acquired by translating the specimen through the light sheet in $200 \mathrm{~nm}$ steps, using a piezo nanopositioning stage (Physik Instrumente, P-621.1CD). The data acquisition program is written in LabVIEW 2013 (National Instruments).

Ampicillin-resistant E. coli K12, constitutively expressing $\mathrm{GFP}^{61}$, were cultured at $37^{\circ} \mathrm{C}$ overnight in LB medium with $100 \mu \mathrm{g} / \mathrm{ml}$ ampicillin. Overnight cultures were diluted 100 times into the same culture medium, grown to an optical density at $600 \mathrm{~nm}$ (OD600) of 0.6-1.0, and then diluted by an additional factor of 10 Round glass coverslips with the diameter of $5 \mathrm{~mm}$ were put into a 24-well plate (Falcon) and $400 \mu \mathrm{l}$ of cell culture was added to the well. Cells were allowed to settle to the bottom of the well and adhere to the coverslip for $1 \mathrm{~h}$. The round coverslips were then mounted onto a sample holder and placed into the LLSM sample-basin filled with M9 medium. GFP fluorescence was excited using $488 \mathrm{~nm}$ light sheet excitation. Biofilm growth was imaged at room temperature every $30 \mathrm{~min}$ for a total of 20 time points. At each time point, a single 3D image stack contained 400 images, each acquired with a $15 \mathrm{~ms}$ exposure time to avoid motion blur.

Myxococcus xanthus strain LS3908 expressing tdTomato under the control of the IPTG-inducible promoter ${ }^{62}$ and DK1622 (WT) were cultured in the nutrientrich CYE media at $30^{\circ} \mathrm{C}$ until it reached an OD600 of 0.6-1.0. Media was supplemented with $1 \mathrm{mM}$ IPTG for tdTomato expressing cells. Chitosan (Thermo Fisher)-coated $5 \mathrm{~mm}$ round glass coverslips were prepared by incubating coverslips with $1 \%(\mathrm{w} / \mathrm{v})$ chitosan $(1.5 \%$ glacial acetic acid $(\mathrm{v} / \mathrm{v}))$ at room temperature for $1 \mathrm{~h}$. Coverslips were then rinsed with water and placed into a 24 -well plate (Falcon) with $350-400 \mu \mathrm{l}$ of undiluted cell culture. WT cells were stained directly in the 24-well plate with $5 \mathrm{ng} / \mathrm{ml} \mathrm{FM4-64} \mathrm{(Thermo} \mathrm{Fisher)} \mathrm{dye.} \mathrm{Cells} \mathrm{were} \mathrm{allowed} \mathrm{to}$ settle and adhere to the coverslip for $2 \mathrm{~h}$. After the settling period, the coverslip was gently rinsed with CYE media to flush away unattached cells. The rinsed coverslip was then mounted onto a sample holder and placed into the LLSM sample-basin filled with MC7 starvation buffer. tdTomato and FM4-64 fluorescence was excited using $561 \mathrm{~nm}$ light sheet excitation. The 3D image stack contained $4002 \mathrm{D}$ images. Each 2D slice was acquired with an exposure time of $30 \mathrm{~ms}$.

For mixed population biofilm imaging, ampicillin-resistant E. coli K12, constitutively expressing GFP ${ }^{61}$, and ampicillin-resistant E. coli $\mathrm{K} 12$, expressing $\mathrm{mScarlet}$ ( $\mathrm{pBAD}$ vector, arabinose induce) were cultured separately at $37^{\circ} \mathrm{C}$ overnight in LB medium with $100 \mu \mathrm{g} / \mathrm{ml}$ ampicillin. Overnight cultures were diluted 100 times into the same culture medium, grown to an optical density at 600 $\mathrm{nm}(\mathrm{OD} 600)$ of $0.6-1.0$, and then diluted to an OD of 0.1. After dilution, the two strains were mixed together. Round glass coverslips with the diameter of $5 \mathrm{~mm}$ were put into a 24-well plate (Falcon) and $500 \mu \mathrm{l}$ of cell culture was added to the well. Cells were allowed to settle to the bottom of the well and adhere to the coverslip for $1 \mathrm{~h}$. The cell culture medium was then removed and replaced by $500 \mu$ M9 medium containing $0.2 \%(\mathrm{w} / \mathrm{v})$ arabinose. The co-culture was incubated at $30{ }^{\circ} \mathrm{C}$ overnight. Ten minutes before imaging, the co-culture was stained with $5 \mathrm{ng} / \mathrm{ml}$ FM4-64 (Thermo Fisher) dye. 3D image stacks of 20 planes with $5 \mathrm{~ms}$ exposure time per frame were acquired using $488 \mathrm{~nm}$ excitation.

Raw data processing. Raw 3D images were background subtracted and then deskewed and deconvolved ${ }^{18,19}$. The background was estimated by averaging intensity values of dark areas (devoid of cells) in the field of view. Deconvolution was performed using the Richardson-Lucy algorithm with ten iterations using experimentally measured point spread functions (PSFs) as the deconvolution kernel. The experimentally measured PSFs were obtained separately for each color channel using fluorescent beads ( $200 \mathrm{~nm}$ FluoSpheres ${ }^{\circledR}$, Thermo Fisher) coated on a coverslip ${ }^{63}$. 3D images were rendered using the $3 \mathrm{D}$ Viewer plugin in Fiji ${ }^{64}$ or ChimeraX $^{65}$

Generation of simulated biofilm images. To generate data for training of CNNs we computationally simulated fluorescence images of 3D biofilms, for which spatial arrangements among individual cells are known precisely and accurately. Growth and division of individual rod-shaped cells in a population were simulated using CellModeller, an individual-based computational model of biofilm growth (Fig. 1a) ${ }^{66}$. In individual-based biofilm growth models, cells are the basic modeling units. Each cell is characterized by a set of parameters, including its $3 \mathrm{D}$ position, volume, and spatial orientation. All the cells in the simulated biofilm are then allowed to evolve in time according to predefined biological, chemical, and mechanical rules. For example, cells grow at a defined rate and then divide after reaching a certain volume threshold. Cellular collisions that are due to cell growth are alleviated by imposing a minimum distance criterion between cells at each time point. For our simulations, we chose cell diameter and cell length $(d, l)$ parameters consistent with a given bacterial species, namely $(1 \mu \mathrm{m}, 3 \mu \mathrm{m})$ for E. coli ${ }^{67},(0.7 \mu \mathrm{m}, 6 \mu \mathrm{m})$ for $M$. xanthus $s^{68}$, and $(1 \mu \mathrm{m}, 1 \mu \mathrm{m})$ 
for spherically symmetric $S$. aureus $^{69}$. While the cell volume can be readily adjusted in CellModeller, the cellular volume density, which is determined by the intercellular spacing, is not directly adjustable. We therefore adjusted the cellular volume density after each simulation by scaling the cellular positions (cell centroids), and thus the intercellular distances by a constant factor, while leaving cell sizes, shapes, and orientations unchanged. This post-processing procedure enabled simulation of the exact same 3D cell arrangements at adjustable cell volume densities.

We fluorescently labeled simulated cell volumes and surfaces according to two commonly used labeling strategies in fluorescence microscopy. To simulate expression of intracellular fluorescent proteins, the fluorescence emitters were placed at random positions within the cell volume. To simulate membrane staining, the fluorescence emitters were placed at random positions on the cell surface. Each cell contained between 500 and 1000 fluorophores to simulate expression level variations between cells, which is often observed in experimental images. Once the fluorophore spatial distributions were determined, a 3D fluorescence image (Fig. 1b) was computationally generated. Each fluorophore was treated as an isotropic point emitter, so that it would produce a diffraction-limited PSF on the detector. Experimentally measured 3D PSF shapes (see "Raw data processing" section) were used as the convolution kernel. Next, the fluorescence signal intensity was scaled by multiplying the image by a constant factor and then a constant background intensity was added to the image at $\sim 200$ photons per pixel, as measured in experimental data. This procedure enabled independent adjustments of the fluorescence signal and background to obtain SBRs consistent with experimental data. In a final step, we introduced Poisson-distributed counting noise, based on the summed background and signal intensities, as well as Gaussiandistributed camera read-out noise (experimentally calibrated for our detector at 3.04 photons per pixel on average) ${ }^{70}$. This resulting image data (Fig. 1c) was then processed in the same manner as experimental data (see "Raw data processing" section). In contrast to experimental data, generation of the corresponding voxellevel annotation maps is fast and error free, because the underlying ground truth cell arrangements are known a priori (Fig. 1d).

To mimic imaging of reporter gene expression in a subset of cells, we simulated biofilm images, in which all cells were stained at the cell surface (e.g., with a membrane-intercalating fluorescent dye) and a subset of cells additionally contained intracellular fluorophores (e.g., through the expression of an intracellular fluorescent protein; Supplementary Fig. S10a, b). The mixing ratios between membrane-labelled, and membrane and interior labelled cells were 10:90, 30:70, 50:50, 70:30, and 90:10. Ten different cell arrangements containing $\sim 300$ cells were simulated for each ratio. To train the CNNs (see next section), six datasets were used, all with a 50:50 mixing ratio.

To mimic imaging of cells with different morphologies, we simulated biofilms containing spherical and rod-shaped cells (Supplementary Fig. S10c, d). Cell arrangements were first simulated using rod-shaped cells and then a fraction of rodshaped cells is replaced with spherical cells. The size of the rod-shaped cells is that of E. coli $(\sim 3 \times 1 \mu \mathrm{m}$, length by diameter). The size of the spherical cells is that of $S$. aureus $(\sim 1 \mu \mathrm{m} \text { in diameter })^{71}$. Both cell types were labelled by intracellular fluorophores, as described above. The mixing ratios between rod-shaped and spherical cells were 10:90, 30:70, 50:50, 70:30, and 90:10. Ten different cell arrangements containing $\sim 300$ cells were simulated for each ratio. To train the CNNs (see next section), we picked one image from each mixing ratio for a total of five images.

Training the convolutional neural networks. We trained 3D U-Net CNNs for voxel-level classification tasks ${ }^{72}$ within the NiftyNet platform ${ }^{73}$ (network architecture depth 4, convolution kernel size 3, ReLU activation function, 32 initial feature maps, and random dropout of 0.5 during training). To achieve robust performance, we trained these networks using five to ten simulated biofilm images with randomly selected cell densities and SBRs (see "Generation of simulated biofilm images" section). The same raw data processing steps used for experimental data (see "Raw data processing" section) were also applied to simulated data. 3D deconvolved simulated data and their corresponding voxel-level annotations were used to train the CNNs. Each image used for training contained $\sim 9$ million voxels. We trained CNNs by classifying each voxel as "background", "cell interior", or as "cell boundary" based on the underlying cell arrangements. For mixed-species biofilms, two additional classes, "cell interior" and "cell boundary" of the second species, were used. This type of annotation scheme has been shown to increase separation of bacterial cells in $2 \mathrm{D}\left(\right.$ ref. $\left.{ }^{74}\right)$. For data augmentation, we applied NiftyNet's built-in scaling, rotation, and elastic deformation functions. Instead of the original cross-entropy loss function combined with uniform sampling, we used the Dice loss function and "balanced sampler", so that every label has the same probability of occurrence in training. All networks were trained for 2000-3600 iterations with a learning rate of 0.0001 . Using these parameters, it took $\sim 24 \mathrm{~h}$ to train the CNNs on a NVIDIA Tesla V100 GPU with 16 GB memory.

Thresholding of CNN-produced confidence maps. Voxel-level classification by CNNs generates different confidence maps (one confidence map for each annotation class). The confidence values range between 0 and 1 , and represent the confidence of assigning individual voxels to a given class. After thresholding the "cell interior" confidence map to obtain a binary image (Supplementary Fig. S11a-c), connected voxel clusters can be isolated and identified as single-cell objects using $3 \mathrm{D}$ connected component labeling ${ }^{75}$. A conservative size-exclusion filter was applied: small objects with a volume approximately ten times less than the expected cell size were considered background noise and filtered out using an area open operator ${ }^{75}$. Since the cell-interior volumes do not contain the cell boundaries, we dilated each object by $1-2$ voxels to increase the cell volumes using standard morphological dilation ${ }^{75}$. The threshold value to segment individual cell objects based on the "cell interior" confidence map was determined by plotting the overall voxel-level segmentation accuracy, quantified as the IoU value (aka Jaccard index ${ }^{76}$ ) vs. the confidence value thresholds (Supplementary Fig. S11d, e). Optimal voxel-level segmentation accuracies were consistently obtained using confidence thresholds between 0.88 and 0.94 . Throughout this work, we use 0.94 for cells labeled with intracellular fluorophores and 0.88 for cells labeled with membranelocalized fluorophores.

Post-processing of U-Net result using a refined LCuts algorithm. Thresholding of the "cell interior" confidence map produces a binary segmentation result (background $=0$, cell interior $=1$ ), where groups of connected, nonzero voxels identify individual cells in most cases. However, when cells are touching, they are often not segmented as individuals, but remain part of the same voxel cluster (undersegmentation). On the other hand, a single cell may be erroneously split into smaller subcellular objects (oversegmentation). Finally, in datasets with low SBR, connected voxel clusters may be detected that do not correspond to cells and thus produce false positive objects (Supplementary Fig. S1a). To address these errors and improve the segmentation accuracy further, we included additional mathematical image analysis steps to post-process the CNN results and reduce undersegmentation and oversegmentation errors.

Step 1: False positive objects are identified by evaluating the coefficient of variation ${ }^{77,78}$ for each connected voxel cluster $i$ :

$$
\mathrm{CV}_{i}=\frac{\sigma_{i}}{\mu_{i}}
$$

where $\sigma_{i}$ and $\mu_{i}$ denote the standard deviation and the mean of the intensity taken over all voxels contained in connected voxel cluster $i$. If the coefficient of variation is larger than $\rho$, then the current object will be classified as a false positive object and removed from the confidence map by setting all its voxels to zero. The removed objects will then no longer be counted when evaluating the cell counting accuracy. The value of $\rho$ is selected based on the coefficient of variation of the background. For the datasets analyzed here, this sample coefficient of variation was determined to be $\rho=1.1$. After CV filtering, objects smaller than $25 \%$ of the expected bacterial cell size are also removed by setting its voxels to zero. The remaining connected voxel clusters are then considered for further processing (Supplementary Fig. S1a).

Step 2: To identify and delineate individual cells in the connected voxel clusters identified in the previous step, we implemented medial axis extraction using the method of inscribed spheres ${ }^{38}$, with the constraint that the sphere radii do not exceed the expected diameter of a single bacterial cell (e.g., $d=0.8 \mu \mathrm{m}$; Supplementary Fig. S1b left). The set of $N$ inscribed spheres are tangent to the object's surface and parameterized by $\left(x_{i}, y_{i}, z_{i} ; r_{i}<d / 2\right)$ for $i=1, \ldots, N$. Determination of the $\left(x_{i}, y_{i}, z_{i} ; r_{i}\right)$ coordinates is achieved using the Euclidean distance transform of the objects' boundary ${ }^{79}$, so that the points with coordinates $\left(x_{i}, y_{i}, z_{i}\right)$ reliably trace out the central cell axes of individual bacterial cells (Supplementary Fig. S1b right).

Step 3: To separate different linear segments after cell axis extraction (Supplementary Fig. S1c), we used a refined version of the LCuts algorithm ${ }^{39,80}$. LCuts is a graph-based data clustering method designed to detect linearly oriented groups of points with certain properties. The fundamental elements of a weighted mathematical graph are nodes, edges, and edge weights. Here, the points with coordinates $\left(x_{i}, y_{i}, z_{i}\right)$ represent the graph nodes. Edges are the connections among nodes. Edges are assigned weights, for example, to reflect the confidence that two nodes belong to the same group. LCuts achieves grouping by assigning weights to edges in the fully connected graph to reflect the similarity between two nodes. The features of each node include its location and direction, where the location of each node is simply its Cartesian coordinates. The direction of each node is found by first determining its 5-hop neighborhood, removing nodes at large relative angles, and evaluating the major direction of the outlier removed neighborhood (Supplementary Fig. S12).

The algorithm to separate the nodes into different groups is a recursive graph cutting method ${ }^{39}$. Graph cuts (e.g., nCut ${ }^{81}$ ) disconnect the edges between two groups of nodes when the combined weights of these edges are minimized. The weights, between node $i$ and node $j$, are calculated as follows:

$$
w_{i j}=w_{D} \cdot w_{T},
$$

where

$$
\begin{gathered}
w_{D}= \begin{cases}e-D_{i j}^{2} / \sigma_{D}^{2} & \text { if } D_{i j}^{2} \leq r \\
0 & \text { if } D_{i j}^{2}>r,\end{cases} \\
w_{T}=e^{-\left(\cos \left(\theta_{i j}\right)-1\right)^{2} / \sigma_{T}^{2}} .
\end{gathered}
$$

$w_{D}$ weighs the distance between two nodes and $w_{T}$ weighs difference between node directions. $D_{i j}$ is the Euclidean distance between node $i$ and node $j$, and $r$ is set 
to eliminate edges between two far away nodes. $\theta_{i j}$ is the relative angle between the directions of nodes $i$ and j. $\sigma_{D}$ and $\sigma_{T}$ are adjustable parameters that control the rate of exponential decay. LCuts continues to separate groups of nodes until each group satisfies a stopping criterion. The stopping criterion is biologically inspired based on the expected length $L$ of a single bacterial cell and a group's linearity after each recursion. LCuts yields linearly oriented groups of points that trace out the central axes of individual cells (Supplementary Fig. S1c). Importantly, cell separation is achieved without having to specify the number of cells in the biofilm in advance. Furthermore, to limit the need for optimization of post-processing routines, the four adjustable parameters used in LCuts, namely cell diameter $d$, the cell length $L$, and the decay parameters $\sigma_{D}$ and $\sigma_{T}$ are chosen based on a priori knowledge about the bacterial cells under investigation. We found that the performance of LCuts is not sensitive to the particular values of $d, L, \sigma_{D}$, and $\sigma_{T}$ as long as they are consistent with the imaged bacterial cell sizes and shapes (Supplementary Fig. S13). Identification of single cells provided by LCuts alleviates undersegmentation errors of the CNN-based segmentation.

Step 4: The final output of linear clustering can provide length, location and orientation of each cell. Based on these linear clusters, the cellular architecture of the biofilms can be reconstructed by placing geometrical models of cells in space, as shown in Supplementary Fig. S1d. For fast computation, spherocylinders are used as the geometrical model using a radius consistent with the known sizes of bacterial cells. To further refine the cell surfaces to better align with the $\mathrm{CNN}$-segmented volumes, we enclosed the inscribed spheres found in Step 2 in a convex hull (Supplementary Fig. S1d).

Performance evaluation. We quantified segmentation accuracy both at the celllevel (object counting) and at the voxel-level (cell shape estimation). To quantify the cell-level segmentation accuracy, we designated segmented objects as true positive (TP) if their voxel overlap with the ground truth or the manual annotation resulted in an IoU value larger than a particular IoU matching threshold. This criterion ensures one-to-one matching. A threshold of 0.5 is typically chosen when reporting single cell counting accuracy values ${ }^{34,40}$. We follow this convention here. If the segmented cell object could not be matched to a ground truth/manually annotated cell volume, then it was counted as a false positive (FP) and the IoU value of that segmented object was set to zero. If a ground truth/manually annotated cell volume was not identified in the image, then it was counted as false negative (FN). The cell (object) counting accuracy was then defined as TP/(TP + $\mathrm{FP}+\mathrm{FN})$. The average IoU value over all segmented objects in the image quantifies the voxel-level segmentation accuracy, i.e., the accuracy of cell shape estimation.

To evaluate the accuracy of cell segmentation on experimental data, three researchers separately traced the cell contours on experimental $2 \mathrm{D}$ slices by using freehand selections in Fiji ROI Manger ${ }^{64}$. Because human annotation is very time consuming $(\sim 50 \mathrm{~h}$ for a complete $3 \mathrm{D}$ dataset containing $\sim 300$ cells in a $22 \times 32 \times$ $12 \mu \mathrm{m}^{3}$ volume), one to three single $2 \mathrm{D}$ slices were selected for each dataset. One exception is the 3D M. xanthus, for which the cell outlines in all available $x$-, $y$-, and $z$-slices were traced manually (Supplementary Fig. S14a). For straight, rod-shaped cells, the centroids of the resulting $2 \mathrm{D}$ cell contours all fall within the cell interior volume. To group together the contours belonging to the same cells, the centroid of each contour was projected along the $x$-, $y$-, and $z$-dimension. If the projected centroid was enclosed by any other contour in a different slice, then the centroid of that contour was projected onto the plane of the initial contour. Two contours were labeled as related if they contained each other's projected centroids (Supplementary Fig. S14b). This process is repeated for all possible contour pairs and their relationship is recorded in an adjacency matrix. Next, related contours were assigned to individual cells (Supplementary Fig. S14c). To separate incorrectly grouped contours, we additionally identified clusters of centroids using the DBSCAN point clustering algorithm ${ }^{82}$ (Supplementary Fig. S14d). In a final step, we manually removed incorrectly traced contours (Supplementary Fig. S14e). Cells are reconstructed by creating convex hulls with the grouped contours (Supplementary Fig. S14f, g). This procedure determined the approximate positions, shapes, and orientations of individual cells in the 3D biofilm.

To estimate the SBRs of both simulated and experimental images, we manually selected and determined the intensities of approximately ten "signal" and ten "background" regions in the images. We computed the SBR as the mean signal intensity divided by the mean background intensity. To estimate the local density of a biofilm, we partitioned the image into several 3D tiles of size 64 by 64 by 8 voxels. We then estimated the local density as the total cell volume contained in each tile divided by the tile volume. We calculated the mean density of the ten densest tiles to define the "local density" metric reported for each dataset in the paper. To estimate the cell density in an experimentally acquired biofilm image, the same calculations were performed on either 3D manual annotations (if available) or binary masks obtained by $\mathrm{CNN}$-processing.

Reporting summary. Further information on research design is available in the Nature Research Reporting Summary linked to this article.

\section{Data availability}

All data used for generating the results presented in this paper are available from the corresponding author upon request. Source data are provided with this paper.

\section{Code availability}

The code for running all the modules of $\mathrm{BCM} 3 \mathrm{D}$, as well as training and test data is available at https://github.com/GahlmannLab/BCM3D.git (ref. ${ }^{83}$ ) and https://doi.org/ 10.5281 /zenodo. 4088658

Received: 18 June 2020; Accepted: 26 October 2020;

Published online: 01 December 2020

\section{References}

1. Hall-Stoodley, L., Costerton, J. W. \& Stoodley, P. Bacterial biofilms: from the natural environment to infectious diseases. Nat. Rev. Microbiol. 2, 95-108 (2004)

2. O'Toole, G., Kaplan, H. B. \& Kolter, R. Biofilm formation as microbial development. Annu. Rev. Microbiol. 54, 49-79 (2000).

3. Nadell, C. D., Drescher, K. \& Foster, K. R. Spatial structure, cooperation and competition in biofilms. Nat. Rev. Microbiol. 14, 589-600 (2016).

4. Persat, A., Inclan, Y. F., Engel, J. N., Stone, H. A. \& Gitai, Z. Type IV pili mechanochemically regulate virulence factors in Pseudomonas aeruginosa. Proc. Natl Acad. Sci. USA 112, 7563-7568 (2015).

5. Zhang, Q. et al. Acceleration of emergence of bacterial antibiotic resistance in connected microenvironments. Science 333, 1764-1767 (2011)

6. Donlan, R. M. Biofilms: microbial life on surfaces. Emerg. Infect. Dis. 8, 881-890 (2002)

7. Yan, J. \& Bassler, B. L. Surviving as a community: antibiotic tolerance and persistence in bacterial biofilms. Cell Host Microbe 26, 15-21 (2019).

8. Kovach, K. et al. Evolutionary adaptations of biofilms infecting cystic fibrosis lungs promote mechanical toughness by adjusting polysaccharide production. NPJ Biofilms Microbiomes 3, 1 (2017).

9. Berk, V. et al. Molecular architecture and assembly principles of Vibrio cholerae biofilms. Science 337, 236-239 (2012).

10. Vidakovic, L., Singh, P. K., Hartmann, R., Nadell, C. D. \& Drescher, K. Dynamic biofilm architecture confers individual and collective mechanisms of viral protection. Nat. Microbiol. 3, 26-31 (2018).

11. Stewart, E. J., Ganesan, M., Younger, J. G. \& Solomon, M. J. Artificial biofilms establish the role of matrix interactions in staphylococcal biofilm assembly and disassembly. Sci. Rep. 5, 13081 (2015)

12. Stewart, E. J., Satorius, A. E., Younger, J. G. \& Solomon, M. J. Role of environmental and antibiotic stress on Staphylococcus epidermidis biofilm microstructure. Langmuir 29, 7017-7024 (2013).

13. Yan, J., Sharo, A. G., Stone, H. A., Wingreen, N. S. \& Bassler, B. L. Vibrio cholerae biofilm growth program and architecture revealed by single-cell live imaging. Proc. Natl Acad. Sci. USA 113, E5337-5343 (2016).

14. Drescher, K. et al. Architectural transitions in Vibrio cholerae biofilms at singlecell resolution. Proc. Natl Acad. Sci. USA 113, E2066-E2072 (2016).

15. Hartmann, R. et al. Emergence of three-dimensional order and structure in growing biofilms. Nat. Phys. 15, 251-256 (2019).

16. Gregor, I. \& Enderlein, J. Image scanning microscopy. Curr. Opin. Chem. Biol. 51, 74-83 (2019).

17. Waldchen, S., Lehmann, J., Klein, T., van de Linde, S. \& Sauer, M. Lightinduced cell damage in live-cell super-resolution microscopy. Sci. Rep. 5 , 15348 (2015).

18. Chen, B. C. et al. Lattice light-sheet microscopy: imaging molecules to embryos at high spatiotemporal resolution. Science 346, 1257998 (2014).

19. Li, D. et al. Extended-resolution structured illumination imaging of endocytic and cytoskeletal dynamics. Science 349, aab3500 (2015)

20. Nadell, C. D., Ricaurte, D., Yan, J., Drescher, K. \& Bassler, B. L. Flow environment and matrix structure interact to determine spatial competition in Pseudomonas aeruginosa biofilms. Elife 6, e21855 (2017).

21. Diaz-Pascual, F. et al. Breakdown of Vibrio cholerae biofilm architecture induced by antibiotics disrupts community barrier function. Nat. Microbiol. 4, 2136-2145 (2019)

22. Chang, B. J. et al. Universal light-sheet generation with field synthesis. Nat. Methods 16, 235-238 (2019).

23. Dean, K. M., Roudot, P., Welf, E. S., Danuser, G. \& Fiolka, R. Deconvolutionfree subcellular imaging with axially swept light sheet microscopy. Biophys. J. 108, 2807-2815 (2015)

24. Chakraborty, T. et al. Light-sheet microscopy of cleared tissues with isotropic, subcellular resolution. Nat. Methods 16, 1109-1113 (2019)

25. Qin, B. et al. Cell position fates and collective fountain flow in bacterial biofilms revealed by light-sheet microscopy. Science 369, 71-77 (2020).

26. $\mathrm{Wu}, \mathrm{Y}$. et al. Spatially isotropic four-dimensional imaging with dual-view plane illumination microscopy. Nat. Biotechnol. 31, 1032-1038 (2013).

27. Dunsby, C. Optically sectioned imaging by oblique plane microscopy. Opt. Express 16, 20306-20316 (2008)

28. Voleti, V. et al. Real-time volumetric microscopy of in vivo dynamics and largescale samples with SCAPE 2.0. Nat. Methods 16, 1054-1062 (2019). 
29. Flemming, H. C. \& Wuertz, S. Bacteria and archaea on Earth and their abundance in biofilms. Nat. Rev. Microbiol. 17, 247-260 (2019).

30. York, A. High NA Single-Objective Light-Sheet https://andrewgyork.github.io/ high_na_single_objective_lightsheet/ (2020).

31. Bouchard, M. B. et al. Swept confocally-aligned planar excitation (SCAPE) microscopy for high-speed volumetric imaging of behaving organisms. Nat. Photonics 9, 113 (2015).

32. Ulman, V. et al. An objective comparison of cell-tracking algorithms. Nat. Methods 14, 1141-1152 (2017).

33. Zhang, M. et al. Source code available at https:/github.com/GahlmannLab/ BCM3D.git (2020).

34. Stringer, C., Wang, T., Michaelos, M. \& Pachitariu, M. Cellpose: a generalist algorithm for cellular segmentation. Preprint at https://doi.org/10.1101/ 2020.02.02.931238 (2020).

35. Moen, E. et al. Deep learning for cellular image analysis. Nat. Methods 16, 1233-1246 (2019).

36. Caicedo, J. C. et al. Evaluation of deep learning strategies for nucleus segmentation in fluorescence images. Cytom. Part A 95, 952-965 (2019).

37. Reyer, M. A., McLean, E. L., Chennakesavalu, S. \& Fei, J. An automated image analysis method for segmenting fluorescent bacteria in three dimensions. Biochemistry 57, 209-215 (2018).

38. Choi, H. I., Choi, S. W. \& Moon, H. P. Mathematical theory of medial axis transform. Pac. J. Math. 181, 57-88 (1997).

39. Wang, J. et al. LCuts: linear clustering of bacteria using recursive graph cuts. In 2019 IEEE International Conference on Image Processing (ICIP), 1575-1579 (IEEE, 2019).

40. Weigert, M., Schmidt, U., Haase, R., Sugawara, K. \& Myers, G. Star-convex polyhedra for 3D object detection and segmentation in microscopy. In 2020 IEEE Winter Conference on Applications of Computer Vision (WACV) 36553662 (IEEE, 2020).

41. Liu, J. et al. Metabolic co-dependence gives rise to collective oscillations within biofilms. Nature 523, 550-554 (2015).

42. Prindle, A. et al. Ion channels enable electrical communication in bacterial communities. Nature 527, 59-63 (2015).

43. Humphries, J. et al. Species-independent attraction to biofilms through electrical signaling. Cell 168, 200-209 e212 (2017).

44. Liu, J. et al. Coupling between distant biofilms and emergence of nutrient time-sharing. Science 356, 638-642 (2017).

45. Mitri, S. \& Foster, K. R. The genotypic view of social interactions in microbial communities. Annu. Rev. Genet. 47, 247-273 (2013).

46. Drescher, K., Nadell, C. D., Stone, H. A., Wingreen, N. S. \& Bassler, B. L. Solutions to the public goods dilemma in bacterial biofilms. Curr. Biol. 24, 50-55 (2014).

47. Persat, A. et al. The mechanical world of bacteria. Cell 161, 988-997 (2015).

48. Papenfort, K. \& Bassler, B. L. Quorum sensing signal-response systems in Gram-negative bacteria. Nat. Rev. Microbiol. 14, 576-588 (2016).

49. Kroos, L. Highly signal-responsive gene regulatory network governing Myxococcus development. Trends Genet. 33, 3-15 (2017).

50. Moon, S. et al. Spectrally resolved, functional super-resolution microscopy reveals nanoscale compositional heterogeneity in live-cell membranes. J. Am. Chem. Soc. 139, 10944-10947 (2017).

51. Bramkamp, M. \& Lopez, D. Exploring the existence of lipid rafts in bacteria. Microbiol. Mol. Biol. Rev. 79, 81-100 (2015).

52. Zou, S. B. et al. Loss of elongation factor $\mathrm{P}$ disrupts bacterial outer membrane integrity. J. Bacteriol. 194, 413-425 (2012).

53. Gonelimali, F. D. et al. Antimicrobial properties and mechanism of action of some plant extracts against food pathogens and spoilage microorganisms. Front. Microbiol. 9, 1639 (2018).

54. Parasassi, T., De Stasio, G., d'Ubaldo, A. \& Gratton, E. Phase fluctuation in phospholipid membranes revealed by Laurdan fluorescence. Biophys. J. 57, 1179-1186 (1990).

55. Strahl, H. \& Hamoen, L. W. Membrane potential is important for bacterial cell division. Proc. Natl Acad. Sci. USA 107, 12281-12286 (2010).

56. Weigert, M. et al. Content-aware image restoration: pushing the limits of fluorescence microscopy. Nat. Methods 15, 1090-1097 (2018).

57. Krull, A., Vicar, T. \& Jug, F. Probabilistic Noise2Void: unsupervised contentaware denoising. Preprint at https://arxiv.org/abs/1906.00651 (2019).

58. Krull, A., Buchholz, T.-O. \& Jug, F. Noise2void-learning denoising from single noisy images. In Proceedings of the IEEE Conference on Computer Vision and Pattern Recognition, 2129-2137 (IEEE, 2019).

59. Buchholz, T.-O., Prakash, M., Krull, A. \& Jug, F. DenoiSeg: joint denoising and segmentation. Preprint at https://arxiv.org/abs/2005.02987 (2020).

60. Valm, A. M. et al. Applying systems-level spectral imaging and analysis to reveal the organelle interactome. Nature 546, 162 (2017).

61. Glass, D. S. \& Riedel-Kruse, I. H. A synthetic bacterial cell-cell adhesion toolbox for programming multicellular morphologies and patterns. Cell 174, 649-658 (2018). e616.
62. Cotter, C. R., Schuttler, H. B., Igoshin, O. A. \& Shimkets, L. J. Data-driven modeling reveals cell behaviors controlling self-organization during Myxococcus xanthus development. Proc. Natl Acad. Sci. USA 114, E4592-E4601 (2017).

63. Gao, R. et al. Cortical column and whole-brain imaging with molecular contrast and nanoscale resolution. Science 363, eaau8302 (2019).

64. Schindelin, J. et al. Fiji: an open-source platform for biological-image analysis. Nat. Methods 9, 676-682 (2012).

65. Goddard, T. D. et al. UCSF ChimeraX: meeting modern challenges in visualization and analysis. Protein Sci. 27, 14-25 (2018).

66. Rudge, T. J., Steiner, P. J., Phillips, A. \& Haseloff, J. Computational modeling of synthetic microbial biofilms. ACS Synth. Biol. 1, 345-352 (2012).

67. Reshes, G., Vanounou, S., Fishov, I. \& Feingold, M. Cell shape dynamics in Escherichia coli. Biophysical J. 94, 251-264 (2008).

68. Starruß, J. et al. Pattern-formation mechanisms in motility mutants of Myxococcus xanthus. Interface Focus 2, 774-785 (2012).

69. Harris, L. G., Foster, S. \& Richards, R. G. An introduction to Staphylococcus aureus, and techniques for identifying and quantifying $S$. aureus adhesins in relation to adhesion to biomaterials: review. Eur. Cell Mater. 4, 39-60 (2002).

70. Yan, T., Richardson, C. J., Zhang, M. \& Gahlmann, A. Computational correction of spatially variant optical aberrations in 3D single-molecule localization microscopy. Opt. Express 27, 12582-12599 (2019).

71. Muller, F. D., Schink, C. W., Hoiczyk, E., Cserti, E. \& Higgs, P. I. Spore formation in Myxococcus xanthus is tied to cytoskeleton functions and polysaccharide spore coat deposition. Mol. Microbiol. 83, 486-505 (2012).

72. Çiçek, Ö., Abdulkadir, A., Lienkamp, S. S., Brox, T. \& Ronneberger, O. 3D U Net: Learning Dense Volumetric Segmentation from Sparse Annotation, 424-432 (Springer International Publishing, Cham, 2016).

73. Gibson, E. et al. NiftyNet: a deep-learning platform for medical imaging. Comput. Methods Progr. Biomed. 158, 113-122 (2018).

74. Van Valen, D. A. et al. Deep learning automates the quantitative analysis of individual cells in live-cell imaging experiments. PLoS Comput. Biol. 12, e1005177 (2016).

75. Acton, S. T. Fast algorithms for area morphology. Digit. Signal Process. 11, 187-203 (2001).

76. Jaccard, P. The distribution of the flora in the alpine zone. N. Phytologist 11, 37-50 (1912)

77. Yu, Y. \& Acton, S. T. Speckle reducing anisotropic diffusion. Trans. Img. Proc. 11, 1260-1270 (2002).

78. Tabassum, N., Vaccari, A. \& Acton, S. Speckle removal and change preservation by distance-driven anisotropic diffusion of synthetic aperture radar temporal stacks. Digit. Signal Process. 74, 43-55 (2018).

79. Yan, T. Q. \& Zhou, C. X. A continuous skeletonization method based on distance transform. Comm. Com. Inf. Sc. 304, 251-258 (2012).

80. Wang, J. Source code available at https://github.com/jwang-c/Postprocessing using-LCuts (2020).

81. Shi, J. B. \& Malik, J. Normalized cuts and image segmentation. IEEE Trans. Pattern Anal. Mach. Intell. 22, 888-905 (2000).

82. Ester, M., Kriegel, H.-P., Sander, J. \& Xu, X. A density-based algorithm for discovering clusters a density-based algorithm for discovering clusters in large spatial databases with noise. In Proceedings of the Second International Conference on Knowledge Discovery and Data Mining, 226-231 (AAAI Press, Portland, Oregon, 1996).

83. Zhang, M. et al. Non-invasive single-cell morphometry in living bacterial biofilms. Preprint at https://doi.org/10.5281/zenodo.4088658 (2020).

\section{Acknowledgements}

This work was supported in part by the US National Institute of General Medical Sciences Grant No. 1R01GM139002 (A.G. and S.T.A.) and by a Jeffress Memorial Trust Award in Interdisciplinary Research (to A.G. and S.T.A.). M.Z. and J.W. were supported by a University of Virginia Presidential Fellowship in Data Science. Y.W. was supported in part by a Dean's M.S. Ph.D. Fellowship in Data Science. We thank Lotte SøgaardAndersen, Larry Shimkets, Huiwang $\mathrm{Ai}$, and Ingmar Riedel-Kruse for providing bacterial strains used in this work. We thank Karsten Siller, computational research consultant at the University of Virginia, for help with utilizing the Universities super-computing resources. We thank Knut Dresher and Eric Jelli for providing the segmentation results using the Hartmann et al. algorithm.

\section{Author contributions}

M.Z., J.Z., Y.W., J.W., S.T.A., and A.G. designed research; M.Z., J.Z., Y.W., J.W., and A.M.A. performed research; M.Z., J.Z., Y.W., J.W., S.T.A., and A.G. analyzed data; and M.Z., J.Z., Y.W., J.W., S.T.A., and A.G. wrote the paper.

\section{Competing interests}

The authors declare no competing interests. 


\section{Additional information}

Supplementary information is available for this paper at https://doi.org/10.1038/s41467020-19866-8

Correspondence and requests for materials should be addressed to A.G.

Peer review information Nature Communications thanks Marius Pachitariu, Thorsten Wohland and the other, anonymous, reviewer(s) for their contribution to the peer review of this work. Peer reviewer reports are available.

Reprints and permission information is available at http://www.nature.com/reprints

Publisher's note Springer Nature remains neutral with regard to jurisdictional claims in published maps and institutional affiliations. (c) (i) Open Access This article is licensed under a Creative Commons Attribution 4.0 International License, which permits use, sharing, adaptation, distribution and reproduction in any medium or format, as long as you give appropriate credit to the original author(s) and the source, provide a link to the Creative Commons license, and indicate if changes were made. The images or other third party material in this article are included in the article's Creative Commons license, unless indicated otherwise in a credit line to the material. If material is not included in the article's Creative Commons license and your intended use is not permitted by statutory regulation or exceeds the permitted use, you will need to obtain permission directly from the copyright holder. To view a copy of this license, visit http://creativecommons.org/ licenses/by/4.0/.

(C) The Author(s) 2020 